\title{
Comparisons of Filters for Noise Removal of Cancer Cell Scanning Electron Microscopy Images
}

\author{
M. Shanmugasundaram, \\ Research Scholar, \\ Erode Arts and Science College, Erode 638 009, \\ Tamil Nadu, India
}

\author{
S. Sukumaran, \\ Associate Professor,Phd, \\ Erode Arts and Science College, Erode 638009 ,
Tamil Nadu, India
}

\begin{abstract}
The challenging task of image processing is to reduce noise in image, which helps to improve the image for further process. This paper proposed bilateral filter, the best choice for removing noise as well as preserving edges in cancer cell image. To show the ability of bilateral filter for removing noise, another famous edge preserving filter called anisotropic filter and a popular multi-scale resolution analysis method called curvelet were tested on breast cancer microscopy images. Experimental result shows that bilateral filter is superior among the tested algorithms in terms of removing noise as well as preserving edges.
\end{abstract}

\section{Keywords}

Bilateral, anisotropic, poisson, Gaussian, noise-removal, curvelet transform

\section{INTRODUCTION}

Breast cancer is a malignant tumor that starts in the cells of the breast. A malignant tumor is a group of cancer cells that can grow into (invade) surrounding tissues or spread (metastasize) into distant areas of the body [1]. Invasive (infiltrating) Ductal Carcinoma, Invasive (infiltrating) Lobular Carcinoma, Ductal Carcinoma in Situ (DCIS), Lobular Carcinoma in Situ (LCIS) are some of the common type breast cancer. Medullary Carcinoma, Inflammatory breast cancers, Mucinous Carcinoma, Paget's disease, Papillary Carcinoma, Phyllodes tumor, Tubular Carcinomas, Metaplastic tumors, Adenoid Cystic Carcinomas, Angiosarcoma are less common type of breast cancer. If a patient is suspected breast cancer, he will be referred for further tests. Mammograms, Ultrasound, MRI, CAT scans and PET scans are some of the important tests used for screening, diagnosis and monitoring the patient.

Scanning Electron Microscope Stereoscopy (SEM-S) is used to characterize 3D features of biological specimens. It (SEM) is invented in the year 1930. With the great help of SEM, both non-biological and biological specimens are able to gain a 3D perspective. However, SEM 3D images are projected onto a 2D plane. A software program is required to import these images that automatically detects feature points in different images and derives necessary information from the detected features. The result of this reconstruction obtains numerical information for further process and decision-making. This information includes the roughness profile, areas and volumes of the acquired surface. Removal of noise is one of the significant tasks of preprocessing of SEM images. Noise levels in LC-SEM (or SEM) images are relatively high [8]. Digital image-processing methods have been applied to highresolution SEM images for the improvement of resolution. Because microscope images have an intrinsic limiting resolution, it often makes little sense to use a noisy, high resolution detector for image acquisition. Microscopic images are generally degraded by noise such as additive (Gaussian), multiplicative (Poisson), and an instrumental imaging property includes glare, shading, geometrical distortion, finite resolution. There are many steps involved to develop a cancer cell processing system such as image acquisition, preprocessing, segmentation and classification. Pre-processing is primary step to remove noise, enhance contrast and isolating area of interest. The significant task of preprocessing is to obtain a representation of cancer cells to make a classification and identify the changes by providing the classifier with the information for recognition.

In this article, different filters were used to de-noise underlying breast cancer cells at various stages of dedifferentiation. In cancer, de-differentiation has been linked with a decreased likelihood of survival and a greater chance that the cancer will spread to other organs [8][13]. As shown in the Results section, profound differences in topography were observed as a cancer becomes less like the normal tissue when it came. These findings have implications not only for improving our understanding how breast cancer grows. Section 2 describes various noise removal and edge preserving techniques in details including bilateral, anisotropic and curvelet de-noising. Section 3 gives an overall experiments and its discussion. Finally section 4 provides conclusion of this article.

\section{METHODOLOGIES}

There are three popular edge preserving filtering methods selected to preprocess cancer cell images. They are

1. Bilateral filtering

2. Anisotropic filtering

3. Curvelet de-noising

The performance of the three filters is evaluated and compared by MSE and PSNR. The value of MSE must be low and PSNR must be high in an image.

\subsection{Bilateral Filter}

Bilateral filtering denotes as a combination of domain and range filtering [2]. In this approach, the pixel value of a position $\mathrm{p}(\mathrm{x}, \mathrm{y})$ is replaced by averaging of similar and nearby pixel values. Apart from smoothing an image, it preserves edges in a good manner with the aid of range filters. The idea of this filtering mechanism to smooth as well as preserving edge is very simple. Discontinuous happened in digital images is very slow. When the filter is moving on the smooth region, it acts like a common domain filter. When the center pixel is located on the boundary between two regions, the idea works here. If the center pixel is located in first region, then the average take place in first region and second region is omitted. Similarly the center pixel is replaced by the average of second region when the pixel location is in second region and the first region pixels are ignored.

The domain filter is defined by the equation 
$h(x)=k_{d}^{-1} \int_{\cdot \alpha \cdot \alpha}^{\alpha} \int^{\alpha} \mathrm{f}(\xi) c(\xi, x) d \xi----(1)$

Where $\mathrm{f}$ is input image and $\mathrm{h}$ is output image. Both are multiband. In this filter, the geometric closeness is determined by the center pixel $\mathrm{x}$ and nearby point $\mathrm{E}$. The equation to preserving the DC component for low-pass filtering is defined by

$$
h_{d}(x)=\int_{\cdot \alpha \cdot \alpha}^{\alpha} \int_{\cdot \alpha}^{\alpha} c(\xi, x) d \xi-------(2)
$$

$\mathrm{K}_{\mathrm{d}}$ is constant. The geometric difference between the central and nearby pixel is defined by the function $c(\xi, x)$. Similarly the range filter applied to image $\mathrm{f}(\mathrm{x})$ is defined by

$$
h(x)=k_{r}^{-1} \int_{\cdot \alpha \cdot \alpha}^{\alpha} \int_{\cdot \alpha} \mathrm{f}(\xi) s(\mathrm{f}(\xi), \mathrm{f}(\mathrm{x})) d \xi--(3)
$$

Function $s(\mathrm{f}(\xi), \mathrm{f}(\mathrm{x}))$ calculates pixel similarity between center pixel $\mathrm{X}$ and nearby pixels $\xi$. Thus $S$ operates in the range function and $c$ operates in the domain of $f$. The normalization constant is replaced by

$k_{r}(x)=\int_{\cdot \alpha \cdot \alpha}^{\alpha} \int_{\cdot \alpha}^{\alpha} s(\mathrm{f}(\xi), \mathrm{f}(\mathrm{x})) d \xi-----(4)$

Combining domain and range filter can be defined by

$$
h(x)=k^{-1}(x) \iint \mathrm{f}(\xi) c(\xi, x) s(\mathrm{f}(\xi) \mathrm{f}(\mathrm{x})) d \xi-(5)
$$

The pixel value at $\mathrm{x}$ can be replaced by averaging similar and nearby pixel values. Thus the bilateral filter is provided by combining the domain and range filter.

$$
k(x)=\iint c(\xi, x) s(\mathrm{f}(\xi) \mathrm{f}(\mathrm{x})) d \xi----(6)
$$

\subsection{Anisotropic Filter}

Anisotropic filtering method is developed by persona and malik[12]. It is a powerful technique to preserve edges when smoothing the images. Mathematically it is formulated as a diffusion process and provides smoothing in preference to smoothing across the boundary [11][9][10]. The performance of this filter is estimated by the statistics of noise degradation and edge strengths. The process of stopping smoothing in boundary can be described by the equation

$$
\frac{\partial}{\partial t} u(x, y, t)=\operatorname{div}(c(x, y, t) \cdot \nabla u(x, y, t))---(7)
$$

Where $u, \nabla u$ and $d i v$ represents gradient and divergence operators respectively. $c(x, y, t)$ reduces the isotropic diffusion. In this method, the edges of image is considered as image gradient and $\mathrm{C}=\mathrm{g}(\nabla, \mathrm{U})$, which has some possible choices of binary and exponential functions. The exponential function is

$g(s)=\exp \left(-\frac{|x|^{2}}{k^{2}}\right)-----(8)$
$\mathrm{K}$ is threshold value to remove noise. It is very important task to discredit the equation. It can be done using four nearest neighbors and the laplacian operator

$U_{i, j}^{n+1}=U_{i, j}^{n}+\lambda\left[C_{N} \cdot \nabla_{N} U+C_{S} \cdot \nabla_{S} U+C_{W} \cdot \nabla_{W} U+C_{E} \cdot \nabla_{E} U\right]_{i, j}^{n}----(9)$

The above equation provides the discrete values of the pixels of the image. Where the $\mathrm{C}$ series in the equation presents the 4-neighbors pixel-values of horizontal and vertical pixels such as north, south, west and east location of the center pixel. It follows that

$$
\begin{aligned}
& C_{N}=g\left(\left|\nabla_{N} U_{i, j}\right|\right)---(10) \\
& \nabla_{N} U_{i, j}=U_{i-1, j}-U_{i, j}----(11) \\
& C_{S}=g\left(\left|\nabla_{S} U_{i, j}\right|\right)----(12) \\
& \nabla_{S} U_{i, j}=U_{i+1, j}-U_{i, j}----(13) \\
& C_{W}=g\left(\left|\nabla_{W} U_{i, j}\right|\right)----(14) \\
& \nabla_{W} U_{i, j}=U_{i, j-1}-U_{i, j}----(15) \\
& C_{E}=g\left(\left|\nabla_{E} U_{i, j}\right|\right)----(16) \\
& \nabla_{E} U_{i, j}=U_{i, j+1}-U_{i, j}----(17)
\end{aligned}
$$

and $\lambda$ is a single parameter required for stability. And the $\Delta$ series represents the gradient values of 4-neighbors.

\subsection{Curvelet Transform De-noise}

Curvelet transform is an emerging technology in the series of multi-scale analysis such as wavelet, contourlet, shearlet and DFT. It obeys a multi-scale law: width $\approx$ length $^{2}$. The process of curvelet transform is grouped into subband decomposition, smooth partitioning, renormalization and ridgelet analysis [7][4][6][5]. A series of low-pass and band-pass filters are used to decompose an image into multiple resolution layers. Each and every layer in the structure is made with different frequencies. This is represented by

$$
f \rightarrow\left(p_{o} f, \Delta_{1} f, \Delta_{3} f, \ldots \ldots\right)----(18)
$$

$\mathrm{P}_{0}$ - low pass-filter.

$\Delta_{1}, \Delta_{2}$ - band- pass filters.

The low and high pass filters are used to deal the low and high frequencies of the signal.

Energy preservation and recursive construction are performed by the equations.

$$
\begin{aligned}
& f=p_{o}\left(p_{o} f\right)+\Sigma \Delta_{s}\left(\Delta_{s} f\right)- \\
& \Psi_{2 s}(s)=2^{4 s} \Psi\left(2^{2 s} X\right)----(20)
\end{aligned}
$$

Curvelet transform implements a curvelet subband using a filter bank of wavelet filters. The subband decomposition $\mathrm{f}$ is approximated by wavelet transforms. So $\mathrm{f}$ is decomposed into $\mathrm{S}_{0}, \mathrm{D}_{1}, \mathrm{D}_{2}$, and $\mathrm{D}_{3}$. The equation for decomposing an image 
into subbands using convolution operator $p_{o} f=\phi_{o} \times f$

$$
\Delta_{s} f=\Psi_{2 s} \times f----(21)
$$

Where $\mathrm{P}_{0} \mathrm{f}$ is obtained by the wavelet coefficients $\mathrm{S}_{0}, \mathrm{D}_{1}, \mathrm{D}_{2}$, $\mathrm{D}_{3}$ and $\Delta_{s} f$ is obtained by $\mathrm{D}_{2 \mathrm{~s}}$ and $\mathrm{D}_{2 \mathrm{~s}+1}$.

Curve detection is achieved by dyadic square [5][6], an important process of smooth partitioning is defined by

$Q_{s, k_{1}, k_{2}}=\left[\frac{k_{1}}{2}, \frac{k_{1}+1}{2}\right] \times\left[\frac{k_{1}}{2^{s}}, \frac{k_{2}+1}{2^{s}}\right] \in Q_{s}$

$\mathrm{Q}_{\mathrm{s}}$ represents all dyadic squares of the grid. A window function is used to smooth each and every square.

$h_{Q}=W_{Q} . \Delta_{s} f----(23)$

Each pixel energy is divided between all sampling windows of the grid by

$\sum_{K_{1}, K_{2}} W^{2}\left(x_{1}-k_{1}, x_{2}-k_{2}\right) \equiv 1----(24)$ Once

it is divided, it must be reconstructed by

$\sum_{Q \in Q} W_{Q} \cdot h_{Q}=\sum_{Q \in Q_{s}} W_{Q}^{2} \cdot h=h----(25)$

Parserval relation can be defined as

$\sum_{Q \in Q}\left\|h_{Q}\right\|_{2}^{2}=\sum_{Q \in Q} W_{Q}^{2} h^{2}=$

$\int \sum_{Q \in Q} W_{Q}^{2} \cdot h^{2}=\int h^{2}=\int\|h\|_{2}^{2}$

The Renormalization of each dyadic square is defined by

$g_{Q}=T_{Q}^{-1} h_{Q}----(27)$

Where $\mathrm{T}_{\mathrm{Q}}$ is normalization operator for $\mathrm{Q}$ is defined by

$\left(T_{Q} f\right)\left(x_{1}, x_{2}\right)=2^{s} f\left(2^{s} x_{1}-k_{1}, 2^{s} x_{2}-k_{2}\right)---$

Ridgelet analysis is final step and it is a constant along with the lines

$x_{1} \cos \theta+x_{2} \sin \theta=$ consant ---- (29)

It can be constructed as wavelet analysis in radon domain. Radon transforms singularities are lying down along with line from point singularities. So, Radon transform of an object is represented by integrals of lines. The radon transform can be defined as

$R f(\theta, t)=\int f\left(x_{1}, x_{2}\right) \delta\left(x_{1} \cos \theta+x_{2} \sin \theta-t\right) d x d y$

Basically ridgelet transform is an application of 1D wavelet transform to the slices of radon transform. The ridgelet element is formularized by

$\hat{\rho}_{\lambda}(\xi)=\frac{1}{2}|\xi|^{-\frac{1}{2}}\left(\hat{\psi}_{j, k}(|\xi|) \cdot \omega_{i, l}(\theta)+\hat{\psi}_{j, k}(-|\xi|) \cdot \omega_{i, j}(\theta+\pi)\right)$

Where $\omega_{i, j}$ are periodic wavelets, $\mathrm{i}$ is the angular scale,

$\psi_{j, k}$ are Meyer wavelets and $\mathrm{j}$ and $\mathrm{k}$ are ridgelet scale and location.

\section{EXPERIMENT}

To preserve anatomy details for the images, they are smoothed to delete the noise and non-stationary artifacts. It is very useful to the doctors to make correct medical diagnosis for cancer. The motivation for choosing the bilateral filter is that the noisy image is weighted by both position distance (spatial filter) and the difference of sinogram values (range filter) [2][3][14][15]. Curvelet transform on the other hand is well suited to handle curve discontinues whereas the famous wavelet is suitable to handle line discontinues [1]. Curvelet represents a scaling law: width $=$ length $^{2}$. In addition, curvelets occurs at all scale, location and orientation.

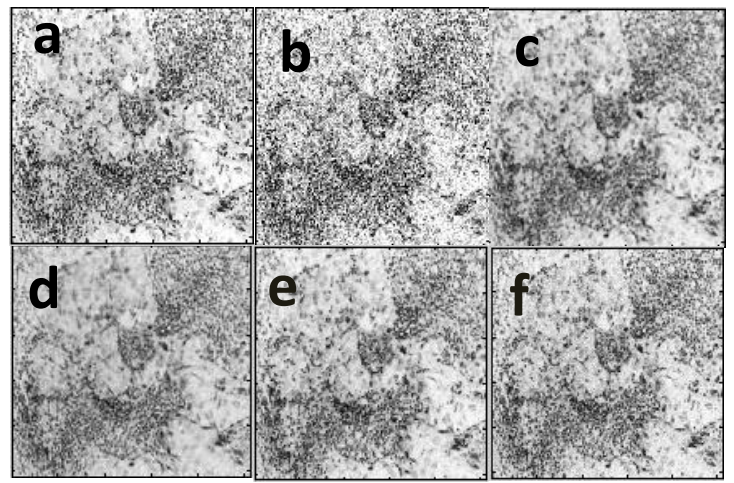

a)original b) Gaussian-noisy c) Gaussian d) curvelet e) anisotropic f) bilateral

Fig 1: Original image and images after applying filters

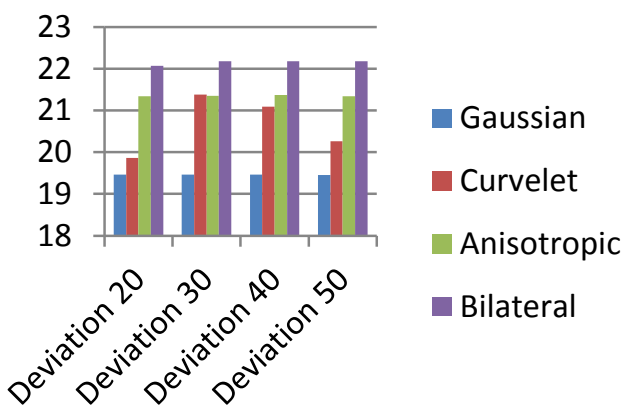

Fig 2: PSNR for Gaussian noise (0.03)

Table 1. PSNR \& MSE values at different noise densityfor Gaussian noise with variance 0.03

\begin{tabular}{|c|c|c|c|}
\hline Method & Density & PSNR & MSE \\
\hline Gaussian & \multirow{4}{*}{20} & 19.468 & 734.97 \\
\hline Curvelet & & 19.861 & 671.37 \\
\hline Anisotropic & & 21.336 & 478.07 \\
\hline Bilateral & & 22.064 & 404.3 \\
\hline Gaussian & \multirow{4}{*}{30} & 19.465 & 735.44 \\
\hline Curvelet & & 21.384 & 472.81 \\
\hline Anisotropic & & 21.351 & 476.42 \\
\hline Bilateral & & 22.177 & 393.93 \\
\hline Gaussian & \multirow{4}{*}{40} & 19.464 & 735.74 \\
\hline Curvelet & & 21.091 & 505.8 \\
\hline Anisotropic & & 21.365 & 474.89 \\
\hline Bilateral & & 22.179 & 393.76 \\
\hline Gaussian & \multirow{4}{*}{50} & 19.45 & 737.97 \\
\hline Curvelet & & 20.261 & 612.27 \\
\hline Anisotropic & & 21.342 & 477.44 \\
\hline Bilateral & & 22.181 & 393.54 \\
\hline
\end{tabular}

To compare the different filters, two cancer cell images has been taken with the size of 512 X512 pixels. Image shown in fig- 1 is degraded by Gaussian noise with 0.03 variance and $50 \%$ density level. To show the robustness of the dominated filter, PSNR and MSE values are calculated at different noise density by the following expression 


$$
P S N R=10 \times \log _{10}\left[\frac{M A X^{2}}{M S E}\right]----(
$$

Where MAX substitutes the maximum pixel value of the tested image.

$$
M S E=\frac{1}{m n} \sum_{i=0}^{m-1} \sum_{j=0}^{n-1}\|x(i, j)-y(i, f)\|^{2}-
$$

Here $\mathrm{x}$ and $\mathrm{y}$ are original and reconstructed images. $\mathrm{m}$ and $\mathrm{n}$ are the number of rows and columns respectively.

Table $1 \& 2$ shows the PSNR and MSE values of filtered images of fig 1 at 20,30, 40 and 50 levels density with 0.03 \& 0.003 variance respectively. Bilateral filter image produces high PSNR values among others.

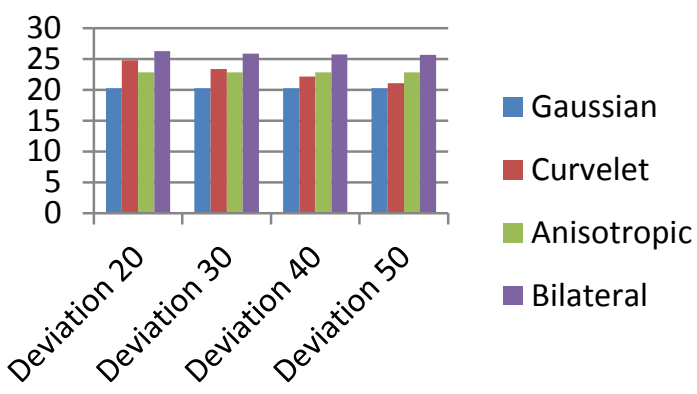

Fig 3: PSNR for Gaussian noise (0.003)

Table 2. PSNR \& MSE values for Gaussian noise

\begin{tabular}{|c|c|c|c|}
\hline Method & Density & PSNR & MSE \\
\hline Gaussian & \multirow{4}{*}{20} & 20.27 & 611.11 \\
\hline Curvelet & & 24.776 & 216.53 \\
\hline Anisotropic & & 22.844 & 337.79 \\
\hline Bilateral & & 26.247 & 154.3 \\
\hline Gaussian & \multirow{4}{*}{30} & 20.265 & 611.82 \\
\hline Curvelet & & 23.371 & 299.21 \\
\hline Anisotropic & & 22.838 & 338.28 \\
\hline Bilateral & & 25.847 & 169.19 \\
\hline Gaussian & \multirow{4}{*}{40} & 20.257 & 612.91 \\
\hline Curvelet & & 22.153 & 396.03 \\
\hline Anisotropic & & 22.832 & 338.73 \\
\hline Bilateral & & 25.712 & 174.54 \\
\hline Gaussian & \multirow{4}{*}{50} & 20.258 & 612.76 \\
\hline Curvelet & & 21.041 & 511.62 \\
\hline Anisotropic & & 22.839 & 338.24 \\
\hline Bilateral & & 25.645 & 177.26 \\
\hline
\end{tabular}
with variance 0.003

In table 3, the de-noised image of poisson noise corrupted in image 1 by bilateral gives a high PSNR value than other three filters. It shows that the bilateral filter is superior among the four to de-noise poisson noise.

Table 3. PSNR \& MSE values for Poisson noise

\begin{tabular}{|l|c|c|}
\hline \multicolumn{1}{|c|}{ Method } & PSNR & MSE \\
\hline Gaussian & 20.255 & 613.22 \\
\hline Curvelet & 21.021 & 513.97 \\
\hline Anisotropic & 22.842 & 337.98 \\
\hline Bilateral & 25.689 & 175.47 \\
\hline
\end{tabular}

With the continuation of this result, additionally all four filters are tested to another image corrupted by poisson noise to show the power of the bilateral filter. Fig- 4 and table 4 shows that once again the bilateral filter dominates other four with respect to PSNR and MSE values. Here the fine details are lost in Gaussian because of over smoothness on the image. Note that the multi-scale curvelet de-noise approach creates curves and wrinkles. Comparing with anisotropic filter, the bilateral filter retrieve the texture and fine details during the process of de-noise.

Table 4. PSNR \& MSE values for Poisson noise

\begin{tabular}{|l|c|c|}
\hline \multicolumn{1}{|c|}{ Method } & PSNR & MSE \\
\hline Gaussian & 22.226 & 389.46 \\
\hline Curvelet & 21.934 & 416.59 \\
\hline Anisotropic & 24.134 & 250.98 \\
\hline Bilateral & 27.377 & 118.94 \\
\hline
\end{tabular}

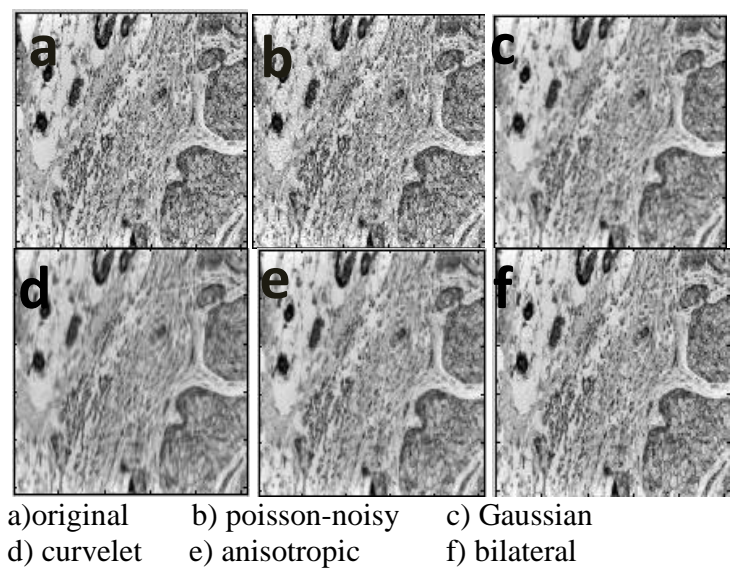

Fig 4: Original image and images after applying filters

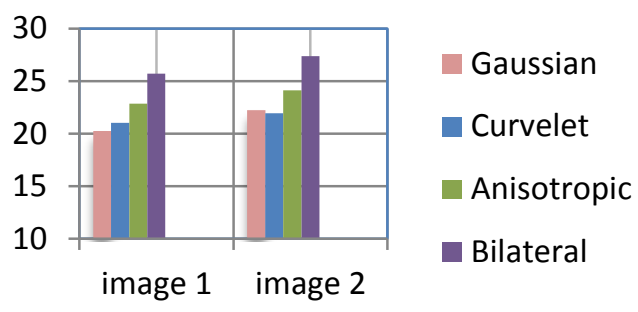

Fig 5: PSNR for Poisson noise

\section{CONCLUSION}

The goal of this article is to evaluate the performance of different de-noising methods to further improve the PSNR. The performance of noise removing with edge preserving was studied by computing error measures on the breast cancer cell test images corrupted by Gaussian and Poisson noises. The parameters considered are the number of variance, PSNR and MSE. Varying the number of filtering iterations, it was found that both edge preserving and noise removal is best in bilateral filter. The de-noising gradually improves the fidelity of feature extraction. The bilateral filter performs better than other for recovering the structured information without losing edges. The other methods will blur edges, unless only a small number of filtering iteration is applied. 


\section{REFERENCES}

[1]. Anil A.Patil, J.Singhai, "Image Denoising Using Curvelet Transform: an approach for edge preservation", Journal of scientific and Industrial Research, vol.69, Jan 2010, pp.34-38

[2]. C. Tomasi and R. Manduchi, "Bilateral Filtering for Gray and Color Images", Proceedings of the 1998 IEEE International Conference on Computer Vision, p 836846, Bombay, India

[3]. D. Barash, "A fundamental relationship between bilateral filtering, adaptive smoothing, and the nonlinear diffusion equation", IEEE Trans. Pattern Anal. Mach. Intell., vol. 24, no.6, p 844-847, Jun. 2002

[4]. D. L. Donoho, "Denoising by Soft-Thresholding", IEEE Transactions on Information Theory 41, 613-627, 1995

[5]. E.Candes, L.Demanet, D.Donoho, L.Ying, "Applied and Computational Mathematics", Department of Stanford university, Stanford, Mar 2006, pp.1-44

[6]. E.J.Candes and D.Donoho, "Curvelet: a surprisingly effective nonadaptive representation for object with edges", Proceeding of Curves and Surfaces IV. France,pp.105-121. 1999

[7]. E.J.Candes, L.Demanet, D.L.Donoho et al., "Fast Discrete Curvelet Transforms. Applied and Computational Mathematics", California Institute of Technology,pp.1 43, 2005

[8]. Eisaku Oho, Norio Baba, Masaru Katoh, Takashi Nagatani, Masako Osumi, KazunobuAmako, and Koichi Kanaya, "Application of the Laplacian filter to highresolution enhancement of SEM images", Journal of Electron Microscopy Technique, Vol. 1,No. 4, pp. 331340, Feb 4 (2005)
[9]. G. Gerig, O. Kubler, R. Kikinis, and F. Jolesz, "Nonlinear anisotropic filtering of MRI data", IEEE Transactions on Medical Imaging, vol. 11, no. 2, pp. 221-232, 1992

[10].M. J. Black, G. Sapiro, D. H. Marimont, and D. Heeger, "Robust anisotropic diffusion," IEEE Transactions on Image Processing, vol. 7, no. 3, pp. 421-432, 1998

[11].N. Nordstrdm, "Biased anisotropic diffusion-A unified regularization and diffusion appraoch to edge detection", Image Vision Comput., vol. 8, no. 4, pp. 318-327, 1990

[12].P. Perona and J. Malik, "Scale space and edge detection using anisotropic diffusion", in Proc. IEEE Workshop Comput. Vision, Miami, FL, Nov. 1987, pp. 16-22

[13].Rebecca K. Anderson, Eric Anderson, LubnaShakir, and Sarah C. Glover, "Image Analysis of Extracellular Matrix Topography of Colon Cancer Cells", MICROSCOPY AND ANALYSIS JULY 2006, page 5-

[14].Wong, Alexander, "Adaptive bilateral filtering of image signals using local phase characteristics", Signal Processing, vol. 88, no. 6, p 1615-1619, June 2008

[15].Zhang Ming, GunturkBahadir, "Multiresolution bilateral filtering for image denoising", IEEE Transactions on Image Processing, vol. 17, no. 12, p 2324-2333, 2008 